\title{
INTEGRAÇÃO DAS ATIVIDADES DE VACINAÇÃO PELO BCG INTRADERMICO NOS PROGRAMAS GERAIS DE IMUNIZAÇÃO DAS UNIDADES SANITARIAS *
}

Roberto Brólio**

Stella Maria C. Nardy**

RSPU-B $/ 303$

BRólio, R. \& NARDY, S. M. C. - Integração das atividades de vacinação pelo $B C G$ intradérmico nos programas gerais de imunização das unidades sanitárias. Rev. Saúde públ., S. Paulo, 10:111-6, 1976.

Resumo: A imunização pelo $B C G$ constitui um recurso operacional de grande alcance nos países em desenvolvimento, onde é elevada a incidência da tuberculose. Os conhecimentos adquiridos nos ültimos cinco anos, em nosso meio, permitem elaborar programas para a aplicação do BCG, por via intradérmica, em extensão útil epidemiologicamente, sem o teste tuberculínico prévio. - analisada a experiência da integração da vacinação pelo $B C G$ intradérmico no programa geral de imunização do Centro de Saude "Geraldo de Paula Souza", da Faculdade de Saúde Pública da USP. As suas conclusões são favorâveis $\grave{a}$ viabilidade do método, permitindo ampla cobertura de imunização anti-tuberculose da população, desde os primeiros meses de vida.

UNITERMos: Vacinação BCG. BCG intradérmico. Imunização, programas.

\section{N T RODU C A O}

A história da vacinação pelo BCG no Brasil tem sido marcada por fases de vacilação, inércia ou incredulidade por parte de profissionais da saúde e de administradores sanitários.

Desde 1927, quando foi realizada a primeira vacinação pelo BCG entre nós, somente agora está se generalizando o conceito de que a mesma deve ser feita, como medida prioritária, pela sua eficácia e rentabilidade em relação ao custo $\mathrm{e}$ benefício que pode proporcionar no combate à tuberculose.

Como fator negativo para a experiência brasileira de vacinação, como vinha sendo feita, exclusivamente por via oral até 1970, faltam elementos comprobatórios de sua eficácia em Saúde Pública, mesmo porque, durante os anos em que foi administrada, não causou nenhum impacto na curva epidemiológica da tuberculose, em nosso meio ${ }^{12}$.

* Trabalho realizado no Departamento de Epidemiologla da Faculdade de Saúde Pública da USP. Apresentado ao I Congresso Brasileiro de Pneumologia e Tísiologia; II Jornada Internacional de Pneumologia e XVII Congresso Nacional de Tuberculose e Doenças Respiratorias, Brasilla, 1975.

** Do Departamento de Epidemiologia da Faculdade de Saúde Pública da USP - Av. Dr. Arnaldo, 715 - São Paulo, SP - Brasil. 
BROLIO, R. \& NARDY, S. M. C. - Integração das atividades de vacinação pelo BCG intradérmico nos programas gerais de imunização das unidades sanitárias. Rev. Saúde públ., s. Paulo, 10:111-6, 1976.

As falhas que podem ser atribuídas à vacinação não se referem certamente à qualidade ou à falta de disponibilidade de vacina, mas aos programas de vacinação que não tiveram a profundidade, continuidade e extensão, em nível nacional, para que pudessem dar os resultados realmente esperados.

A vacina brasileira que teve em Arlindo de Assis um dos seus mais ardorosos defensores e que é considerada de boa qualidade pelos técnicos da Organização Mundial da Saúde (OMS) ${ }^{3,10}$, é produzida pela Fundação "Ataulfo de Paiva" do Rio de Janeiro, e pode ter sua produção facilmente incrementada em quantidade suficiente para vacinar toda a população suscetivel do Brasil.

A OMS recomenda a vacinação como recurso operacional de linha de frente, contra a tuberculose, nos países onde a moléstia constitui problema de saúde públicá ${ }^{11}$.

Além de ensaios controlados que comprovam sua eficácia no homem, dos quais a experiência inglesa é referida como exemplo ${ }^{9}$, existem trabalhos que evidenciam sua viabilidade como medida de saúde pública ${ }^{1, ~}{ }^{7}$. O Japão em 1946, ao final da segunda guerra mundial, tinha a tuberculose como um dos principais problemas sanitários a exigir solução, e a vacinação, instituída como medida compulsória, comprovou sua eficácia entre os demais recursos operacionais, pelo seu elevado efeito epidemiológico em relação ao custo dispendido ${ }^{1}$.

A Divisão Nacional de Tuberculose ${ }^{6}$ (DNT) que planeja, supervisiona e coordena as atividades de luta anti-tuberculose no País, programou um vasto plano de vacinação para ser executado até 1979, visando fazer a vacinação pelo BCG intradérmico (BCG-id) dos jovens de 5 a 15 anos e, havendo viabilidade, também dos recém-nascidos e menores de 5 anos.

É necessário, todavia, que o programa tenha continuidade e possa alcançar todas as faixas da população suscetível de adquirir a infecção tuberculosa.

O presente trabalho analisa as atividades de vacinação anti-tuberculose no Centro de Saúde "Geraldo de Paula Souza" da Faculdade de Saúde Pública da USP (CS-LSP) e tem por fim evidenciar a viabilidade do método e as vantagens que podem advir da integração da vacinação pelo BCG-id como rotina, nas atividades gerais de imunização das unidades sanitárias.

\section{ATIVIDADES DE IMUNIZAÇĀO}

O Departamento de Epidemiologia da Faculdade de Saúde Pública da LSP na sua área de Tisiologia vem desenvolvendo um programa de vacinação pelo BCG-id, em nosso meio, desde 1970, quando realizou um ensaio controlado ${ }^{t}$ e a seguir, em 1971, iniciou o programa de imunização de escolares ${ }^{5}$.

Em janeiro de 1975 a vacinação foi introduzida como atividade isolada na área de tisiologia do CS-LSP para atender à demanda da população e aos encaminhamentos de médicos interessados na vacinação.

A partir de agosto desse mesmo ano, a vacinação pelo BCG-id foi incorporada às atividades de vacinação do CS-L'SP, como rotina, para as pessoas que são atendidas na referida unidade sanitária. A vacinação pelo BCG-id que vinha sendo feita como atividade de atendimento especializado no setor de tisiologia, passou a integrar as atividades gerais de imunização, e o BCG-id passou a constar no calendário de vacinação ao lado das demais vacinas. 
BROLIO, R. \& NARDY, S. M. C. - Integração das atividades de vacinação pelo BCG intradérmico nos programas gerais de imunização das unidades sanitárias. Rev. Saúde públ., S. Paulo, 10:111-6, 1976

O programa de vacinação foi elaborado para dar atendimento às crianças desde o segundo mês de vida.

Após ser feita a aplicação de outra vacina, a criança é encaminhada para a aplicação do BCG. Antes da vacinação, a mãe ou responsável recebe uma explicação, feita pela educadora, sobre a eficácia da vacina e as possíveis reações e duração das lesōes vacinais.

A vacinação é indiscriminada, sem a aplicação prévia do teste tuberculínico.

O BCG é aplicado por via intra-dérmica, com agulha e seringa, no braço direito, no local de inserção inferior do músculo deltoide, na quantidade de $0,1 \mathrm{ml}$, contendo $0,1 \mathrm{mg}$ de bacilos-vacina.

O BCG empregado é o liofilizado, produzido pela Fundaçáo "Ataulfo de Paiva" do Rio de Janeiro e fornecido pela Divisão Nacional de Tuberculose (DNT).

As seringas $e$ agulhas utilizadas na aplicação do BCG são padronizadas e fornecidas pela DNT.

A equipe de trabalho, previamente treinada para a aplicação do BCG, observa rigorosamente as normas e técnicas recomendadas pela DNT para a aplicação da vacina.

O controle das lesões vacinais é feito pela supervisora de aplicação do BCG e pelos médicos do setor, tanto para esclarecimentos e orientação, como para o acompanhamento das cicatrizações das lesóes vacinais e assistência, quando necessária.

\section{COMENTARIOS}

A vacinação pelo $B C G$-id vem sendo realizada normalmente, sem prévia aplicação do teste tuberculínico, dentro de um perfeito entrosamento de Serviços, e tem tido boa aceitação por parte do público e dos profissionais da saúde.

0 programa foi planejado para ter continuidade, e se espera que possa produzir uma proteção efetiva da população vacinada, na área de atendimento do CS-USP.

A medida reveste-se de um alto alcance sanitário e pode ser adotada como modelo para a imunização contra a tuberculose, em outras unidades destinadas a dar assistência integral no setor saúde.

Para Payne ${ }^{13}$, a vacinação pelo $B C G$, integrada nos programas gerais de imunização, constitui o recurso operacional indicado para dar uma cobertura ampla e contínua de vacinação, capaz de produzir resultados significativos na curva epidemiológica da tuberculose.

A vacinação pelo BCG, através de programações verticais ou de campanhas a curto prazo, deve ser considerada como recurso de emergência, destinado a reduzir a magnitude do problema, para que os serviços integrados possam manter, de maneira permanente e a longo prazo, a continuidade de imunização do maior número possível de pessoas.

Não há dúvida que os sistemas não se excluem. Na prática os dois sistemas estão estreitamente relacionados. 0 sistema vertical não produzirá efeito duradouro se não for complementado por atividades no sistema horizontal, numa base permanente e contínua.

Admite-se que a vacina brasileira seja dotada de capacidade de imunização equivalente à da vacina inglesa, cuja eficácia se eleva a $80 \%$ depois de 15 anos da aplicação ${ }^{\circ}$. Para que a vacina possa dar resultados significativos na curva epidemiológica da tuberculose, deve ser feita em nível de cobertura superior a $80 \%$ das pessoas não reatoras a fim de garantir 
BROLIO, R. \& NARDY, S. M. C. - Integração das atividades de vacinação pelo BCG intradérmico nos programas gerais de imunização das unidades sanitárias. Rev. Saúde públ., S. Paulo, 10:111-6, 1976.

uma proteção mínima de $64 \%$ da população suscetível de adquirir a infecção natural.

Como afirma Gironda ${ }^{8}$, a prática da vacinação em larga escala pelo BCG-id constitui a meta prioritária e eficaz, especialmente nos países em desenvolvimento onde a endemia tuberculosa é elevada.

A vacinação indiscriminada proporciona vantagens operacionais, pois em certas áreas, como nos escolares, a aplicação da vacina apenas nas crianças não reatoras levaria a uma perda média de $20 \%$ de vacinações, considerando-se os não comparecimentos de crianças ao estabelecimento de ensino, nos dias de aplicação e da leitura do teste tuberculínico:2. E se a vacinação não for feita no mesmo dia da leitura do teste, as perdas serão maiores.

A aplicação da vacina após o teste tuberculínico apresenta menor rendimento e exige um considerável esforço operacional, incompatível com a realização de programas de massa. Contudo, o melhor efeito epidemiológico da vacinação é obtido com a aplicação da vacina nos não infectados, pois a mesma tem por fim desenvolver na criança uma infecção benigna, capaz de evitar o risco de uma infecção natural potencialmente grave.

A percentagem de não reatores é maior nos primeiros anos de vida, fase em que a tuberculose é particularmente grave, e em que há maiores riscos de ocorrer disseminações linfo-hematogênicas e a meningite específica.

A oportunidade de vacinação nesta fase da vida reverte-se de particular interesse e pode ser realizada, amplamente, nas unidades sanitárias, através do sistema integrado de imunizações.

Em síntese a integração pode proporcionar as seguintes vantagens:
1 - que a vacinação seja programada para todo o País utilizando a rede de unidades sanitárias, estatais e para-estatais.

2 - que os programas de imunização sejam extendidos, de maneira permanente e contínua, ao major número de pessoas desde os primeiros meses de vida.

3 - que a vacinação seja planejada para dar uma cobertura não inferior a $80 \%$ das pessoas não infectadas especificamente.

4 - que a vacinação possa ser feita sem excluir a possibilidade de realização de campanhas especialmente centradas em certas coletividades, como de escolares e de recém-nascidos.

\section{CONCLUSOES}

As atividades de imunização do CS USP evidenciam a viabilidade do sistema de integração da vacinação pelo BCG-id nos programas gerais de imunização.

As atividades de vacinação anti-tuberculose são de natureza essencialmente preventivas e devem ser centradas, de preferência, nas pessoas ainda não infectadas especificamente.

Atendendo às facilidades operacionais, em nível de Saúde Pública, a vacinação indiscriminada, de infectados e não infectados, é de maior alcance relativamente ao custo e benefício que pode proporcionar.

O sistema integrado permite realizar a imunização de maneira ampla e contínua, desde os primeiros meses de vida, e com maior probabilidade de imunizar as crianças ainda não infectadas, onde os resultados que se espera da vacinação são mais significativos. 
BRoLIO, R. \& NARDY, S. M. C. - Integração das atividades de vacinação pelo BCG intradérmico nos programas gerais de imunização das unidades sanitárias. Rev. Saúde públ., S. Paulo, 10:111-6, 1976.

RSPU-B/303

Brólio, R. \& NARDY, S. M. C. - [Integration of intradermic BCG vaccination in general public health immunization programmes]. Rev. Saúde públ., S. Paulo, 10:111-6, 1976.

SUMmary: Immunization by $B C G$ is of great effect in developing countries where the incidence of tuberculosis is high. The knowledge that has been acquired in our environment in the last five years has made possible the organization of programmes for $B C G$ intradermic immunization, to a useful epidemiological extent, without a prior tuberculin test. The practice of the integration of intradermic $B C G$ vaccination in the general immunization programmes of the "Geraldo de Paula Souza" Health Unit of S. Paulo University School of Public Health was analysed and the conclusion was favourable as to the viability of the method, which allows a large-scale anti-tuberculosis immunization of the population right from the very first months of life.

UNITERMS: $B C G$ vaccination. BCG intradérmico. Immunization, programmes.

\section{REFERENCIAS BIBLIOGRÁFICAS}

1. AZUMA, Y. - BCG vaccination in Japan. Bull. int, un. Tuberc., 2:157-61, 1972 .

2. BRólIO, R. et al. - Programa desenvolvido na pesquisa da sensibilidade tuberculinica e vacinação pelo BCG intradérmico, em escolares do primeiro ano da Rede Municipal de Ensino de São Paulo, durante o ano de 1971. Rev. Div. nac. Tuberc., 18: $46-52,1974$.

3. BUNCH-CHRISTENSEN, K. et al. - The virulence of some strains of BCG for golden hamsters. Bull. Wld. Hlth. Org., 43:65-70, 1970 .

4. CERTAIN, D. A. et al. - Ensaio de BCG intradérmico em escolares de São Paulo, Capital, 1970. Rev. Div. nac. Tuberc., 15:281-7, 1971.

5. CERTAIN, D. A. et al. - Análise dos resultados da pesquisa da infecção tuberculosa e do primeiro programa de vacinação pelo $\mathrm{BCG}$ intradérmico em escolares de São Paulo, Brasil, 1971-1974. Rev. Saude pübl., S. Pau10, $9: 125-36,1975$.

6. DIVISAO NACIONAL DE TUBERCULOSE. Comissão de BCG: vacinação
BCG no Brasil. Rev. Div, nac. Tuberc., 17:255-62, 1973.

7. GERNEZ-RIEUX, C. \& GERVOIS, M. Protection conférée par le BCG pendant les vingt années suivant la vaccination. Bull. Wld. Hlth. Org., 48:139-54, 1973.

8. GIRONDA, L. C. - Integración de la vacunación $B C G$ en los programas generales de imunización. (Apresentado ao Congresso Panamericano de la Tuberculosis y Enfermedades del Aparato Respiratório XVIII, e Congresso Venezolano de Tisiologia y Neumologia, VII, Caracas, Venezuela, 1974).

9. GRÃ-BRETANHA. Medical Research Council. BCG and vole bacillus vaccines in the prevention of tuberculosis in adolescence and early adult life: fourth report. Bull. Wld. Hith. Org., 46:371-85, 1972.

10. LADEFOGED, A. et al. - The protective effect in bank voles of some strains of BCG. Bull. Wld. Hlth. Org., 43: T1-90, 1970. 
BRóLIO, R. \& NARDY, S. M. C. - Integração das atividades de vacinação pelo BCG intradérmico nos programas gerais de imunização das unidades sanitárias. Rev. Saúde públ., S. Paulo, 10:111-6, 1976

11. ORGANIZAÇÃO MUNDIAL DA SAÚdE. Comitê de Expertos de la OMS en Tuberculosis, 9.0, Ginebra, 1973. Informe... Ginebra, 1974. (Ser. Inf. tecn., 552).

12. PAULA SOUZA, R. de - Valor da orovacinação $B C G$ do recém-nascido. Rev. Div. nac. Tuberc., 17:61.71, 1973 .
13. PAYNE, A. M. M. - Sistemas de lucha contra las enfermedades transmissibles. Cron. Org. mund. Salud, 22: 3-8, 1968 ,

Recebido para publicaşão em $05 / 12 / 19 \% 5$

Aprovado para publicacáo em $05 / 01 / 19 \% 6$ 